\title{
BMJ Open Protocol for a quasi-experimental study to assess the feasibility, acceptability and costs of multiple first-lines artemisinin-based combination therapies for uncomplicated malaria in the Kaya health district, Burkina Faso
}

\author{
Mohamadou SIRIBIE (D) , ${ }^{1}$ André-Marie Tchouatieu, ${ }^{2}$ Issiaka Soulama, ${ }^{1}$ \\ Jean Moise Tanga Kaboré, ${ }^{1}$ Yacouba Nombré, ${ }^{3}$ Denise Hien, ${ }^{1}$ Alice Kiba Koumaré, ${ }^{3}$ \\ Nouhoun Barry, ${ }^{1}$ Adama Baguiya (D) , ${ }^{4}$ Alimatou Héma, ${ }^{1}$ Frédéric Dianda, ${ }^{3}$ \\ Yacouba Savadogo, ${ }^{3}$ Seni Kouanda, ${ }^{5}$ Alfred Bewendtaoré Tiono, ${ }^{1}$ \\ Sodiomon Bienvenu Sirima ${ }^{1}$
}

To cite: SIRIBIE M, Tchouatieu A-M, Soulama I, et al. Protocol for a quasiexperimental study to assess the feasibility, acceptability and costs of multiple first-lines artemisinin-based combination therapies for uncomplicated malaria in the Kaya health district, Burkina Faso. BMJ Open 2021;11:e040220. doi:10.1136/ bmjopen-2020-040220

- Prepublication history and supplemental material for this paper is available online. To view these files, please visit the journal online (http://dx.doi. org/10.1136/bmjopen-2020040220).

Received 08 May 2020 Revised 22 September 2020 Accepted 16 December 2020

Check for updates

(C) Author(s) (or their employer(s)) 2021. Re-use permitted under CC BY-NC. No commercial re-use. See rights and permissions. Published by BMJ.

For numbered affiliations see end of article.

Correspondence to Dr Mohamadou SIRIBIE; m.siribie@gmail.com

\section{ABSTRACT}

Introduction As demonstrated in mathematical models, the simultaneous deployment of multiple firstline therapies (MFT) for uncomplicated malaria, using artemisinin-based combination therapies (ACTs), may extend the useful therapeutic life of the current ACTs. This is possible by reducing drug pressure and slowing the spread of resistance without putting patients' life at risk. We hypothesised that a simultaneous deployment of three different ACTs is feasible, acceptable and can achieve high coverage rate if potential barriers are properly identified and addressed.

Methods and analysis We plan to conduct a quasiexperimental study in the Kaya health district in Burkina Faso. We will investigate a simultaneous deployment of three ACTs, artemether-lumefantrine, pyronaridineartesunate, dihydroartesinin-piperaquine, targeting three segments of the population: pregnant women, children under five and individuals aged five years and above. The study will include four overlapping phases: the formative phase, the MFT deployment phase, the monitoring and evaluation phase and the post-evaluation phase. The formative phase will help generate baseline information and develop MFT deployment tools. It will be followed by the MFT deployment phase in the study area. The monitoring and evaluation phase will be conducted as the deployment of MFT progresses. Cross-sectional surveys including desk reviews as well as qualitative and quantitative research methods will be used to assess the study outcomes. Quantitatives study outcomes will be measured using univariate, bivariate and multivariate analysis, including logistic regression and interrupted time series analysis approach. Content analysis will be performed on the qualitative data.

Ethics and dissemination The Health Research Ethics Committee in Burkina Faso approved the study (Clearance no. 2018-8-113). Study findings will be disseminated through feedback meetings with local communities,

\section{Strengths and limitations of this study}

Theoretical models have shown that the simultaneous deployment of artemisinin-based combination therapies (ACTs) for the management of uncomplicated malaria cases may extend the useful therapeutic life of the current ACTs by reducing drug pressure and slowing the spread of resistance without putting patients' lives at risk.

- This quasi-experimental study aims at assessing the feasibility, acceptability and costs of multiple firstline therapies (MFT) deployment with three ACTs targeting three population segments for the management of uncomplicated malaria.

- This study will provide evidence for policymakers to facilitate the rapid uptake of the strategy aiming at preventing the emergence and spread of Plasmodium falciparum resistance to ACTs in malaria endemic areas.

- The study will not compare changes in molecular markers of resistance to ACTs before and after the deployment of the MFT.

national workshops, oral presentations at congresses, seminars and publications in peer-reviewed scientific journals.

Trial registration number NCT04265573.

\section{INTRODUCTION}

Despite coordinated control efforts, malaria remains the leading cause of morbidity and mortality in Burkina Faso. Its transmission is intense and perennial but peaks during the rainy season. In 2018, a total of $11,463,808$ uncomplicated malaria cases, 506,513 severe 
malaria cases and 4,294 malaria imputable deaths were recorded in all health facilities in Burkina Faso. Malaria was then responsible for $41.3 \%$ and $25.9 \%$ of outpatients visits at peripheral health facilities and medical centres/hospitals, respectively. In peripheral health facilities, $57 \%$ of all-causes hospital admission and $36.3 \%$ of all-causes deaths were associated with malaria. ${ }^{1}$ The main malaria vectors are Anopheles gambiae, Anopheles arabiensis and Anopheles funestus. ${ }^{2}$

Malaria control strategy in Burkina relies on an integrated approach combining (1) vector control tools, (2) chemoprevention in vulnerable groups (intermittent preventive treatment during pregnancy and the seasonal malaria chemoprevention (SMC) in children under five); and (3) prompt and adequate malaria case management both at community level (Community Case Management of malaria) and health facility level. ${ }^{2}$

As per the World Health Organization (WHO) recommendation, all cases of clinical malaria in Burkina Faso should be biologically confirmed with a malaria rapid diagnostic test (mRDT) or microscopy, when available, before any treatment. The National Malaria Control Programme (NMCP) guidelines recommend the following three artemisinin-based combination therapies (ACTs), amodiaquine-artesunate (AQ-AS) or artemether-lumefantrin (AL) or dihydroartemisinin-piperaquine (DHA-PQ) as first-line drugs for the treatment of uncomplicated malaria. ${ }^{3}$ WHO prequalified pyronaridine-artesunate (PYR-AS) granules for oral suspension is registered in Burkina Faso and available at private pharmacies across the country. Several studies conducted in sub-Saharan Africa, including Burkina Faso, demonstrated the safety and the efficacy of these four ACTs (AS-AQ AL, DHA-PQ and PYR-AS) against uncomplicated malaria. ${ }^{4-7}$ In addition to oral quinine, ACT is recommended for the management of uncomplicated malaria cases in pregnant woman except during the first trimester of pregnancy. Four ACTs (DHA-PQ AL, AS-AQ and AS-mefloquine) shown to be well-tolerated and effective in pregnant women in sub-Saharan Africa, including Burkina Faso, as reported by the Safety and efficacy of four artemisinin-based combination treatments in African pregnant women with malaria (PREGACT) Study group. ${ }^{8}$

One of the main threats to malaria control and elimination is the emergence and the spread of artemisinin-resistant Plasmodium falciparum parasites. Artemisinin resistance, first reported in Western Cambodia, is characterised by slow parasite clearance, ${ }^{910}$ increased ACTs treatmentfailure rates, ${ }^{11}$ and it has linked recently with point mutations in the 'propeller' region of a P. falciparum Kelch protein. ${ }^{12}$ Artemisinin resistance has since spread, emerged independently, or both in other areas of mainland Southeast Asia. ${ }^{13-18}$ To address this real threat, different approaches have been proposed or are being developed, such as the research and development of new antimalarial agents, ${ }^{19}$ the concept of triple combination therapy in which two slowly eliminated partner drugs are combined with an artemisinin derivative,${ }^{20}$ and the use of multiple first-line therapies (MFT) combining drugs with different or opposing selection pressures. ${ }^{21}$ Although current ACTs still remain effective across sub-Saharan
African countries, new strategies optimising their use must be promoted as the development of new alternatives drugs is progressing slowly.

In this study, we will evaluate to what extent the MFT strategy is feasible and acceptable. We will also document the cost of its deployment to the health system.

\section{METHODS AND ANALYSIS \\ Study site}

The study will be conducted in the Kaya health district. In 2007, the 'Institut de Recherche en Sciences de la Santé' (IRSS) created a research platform, the Kaya Health and Demographic Surveillance System (Kaya-HDSS). ${ }^{22}$ The Kaya health district is located in the north-central region, $100 \mathrm{~km}$ from Ouagadougou, the capital of Burkina Faso. It covers one urban and three rural municipalities, with a population estimated at 407311 inhabitants in 2018. ${ }^{1}$ The district has forty (40) first-level public health facilities, including 39 primary healthcare centres and one medical centre with a surgery unit. In addition, there are four private and confessional health facilities. The health district includes the regional hospital of Kaya, which is the referral hospital for the northcentral region. In 2018, 191771 uncomplicated malaria cases were recorded in the district. In 2018, the malaria incidence was 1.27 per person-year among children under five, and 0.4 per person-year among the overall population. The number of malaria cases treated with ACTs was 188527 in the same year. ${ }^{1}$

In the Kaya district, healthcare is provided by the primary health centres, the medical centre and the regional hospital centre. Outpatient and inpatient services, vaccination services, child healthcare and antenatal care services are available 24 hours a day and 7 days a week. All health centres have medicines dispensing facility, distributing mainly generic drugs. A free healthcare policy has been in place in Burkina Faso since April 2016 for children under five and pregnant women.

\section{Study design}

This is a quasi-experimental study aiming at assessing the feasibility, acceptability and costs of a MFT programme for uncomplicated malaria. The programme will include four overlapping phases: the formative phase, the MFT deployment phase, the monitoring and evaluation phase and the post-evaluation phase, illustrated in figure 1. A mixedmethod, including desk reviews, quantitative and qualitative surveys will be conducted to assess the programme outcomes. The programme is anticipated to last 22 months, from 2019 to 2021.

Three ACTs will be deployed at the health facility level. Each of them will be used for the management of uncomplicated malaria in one of the following population groups: PYR-AS for children under five, AL for pregnant women and DHA-PQ for individuals aged five years or above. Community case management of malaria $(\mathrm{CCMm})$ will be maintained in compliance with current NMCP recommendations, with AL given across all age categories but not to pregnant 


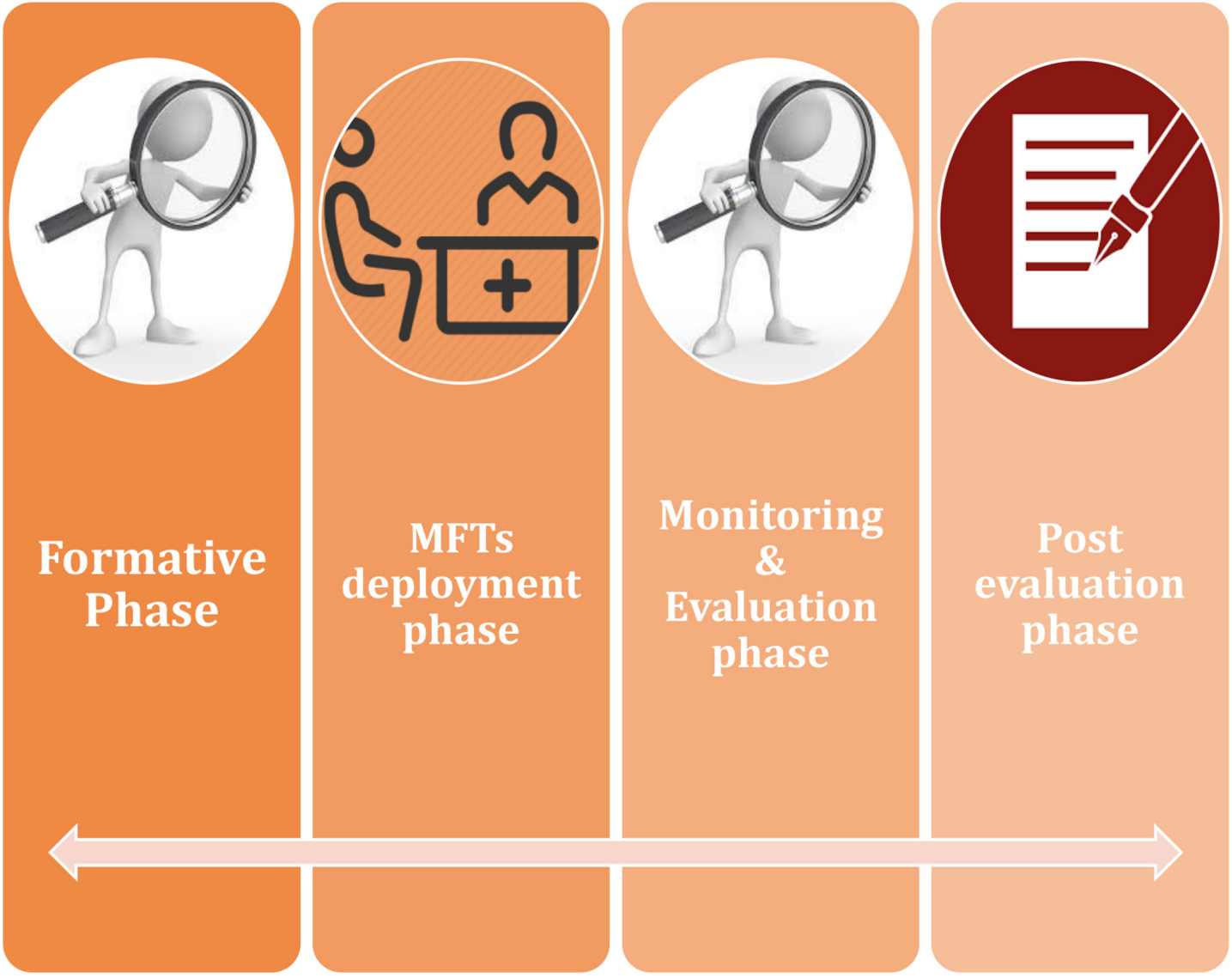

Figure 1 Design of the programme. MFT, multiple first-line therapies.

women. Sufficient stocks of ACTs will be secured for the duration of MFT deployment to avoid both stock-outs and drug expiry. The ACTs supply chain will comply with the official drugs distribution channel illustrated in figure 2. No parallel supply chain will be permitted. All members involved in the implementation of the MFT programme will be identified and provided appropriate training on their role and responsibilities. The inclusion and non-inclusion criteria for the three study's ACTs are as follows:

- For PYR-AS: inclusion criteria are children under five and weighing at least $5 \mathrm{~kg}$. The non-inclusion criterion is the know hypersensitivity to a component of PYR-AS.

- For DHA-PQ: inclusion criterion is individuals at least five years old and non-inclusion criterion is the know hypersensitivity to a component of DHA-PQ.

- For AL: inclusion criterion is pregnant woman and non-inclusion criteria are first trimester of pregnancy and know hypersensitivity to a component of AL.

\section{Study objectives}

The objectives of the different phases of the programme are as follows:

\section{Formative phase}

- General objective:

To generate baseline information and develop intervention tools for the MFT programme for the management of uncomplicated malaria.

- Specific objectives:
- To consolidate the programme working group.

- To assess the perceptions and expectations regarding the MFT programme of the Heath system's key stakeholders and the community members.

- To document any perceived or existing obstacles/threats to the implementation of the MFT programme.

- To assess the treatment-seeking behaviour for febrile episodes/malaria.

- To assess the morbidity and the mortality related to febrile episodes/malaria.

- To develop a training manual as well as promotional and educational tools to optimise the programme's implementation and uptake.

- To develop tools for the monitoring and evaluation of the MFT programme implementation.

\section{MFT deployment phase}

- General objective:

To implement the MFT for the management of uncomplicated malaria cases that is feasible, acceptable and able to achieve a high coverage rate.

- Specific objectives:

- To train and raise awareness among the key stakeholders/implementers of the MFT programme.

- To ensure continuous availability of study medicines delivered through the official drug supply chain system in Burkina Faso. 


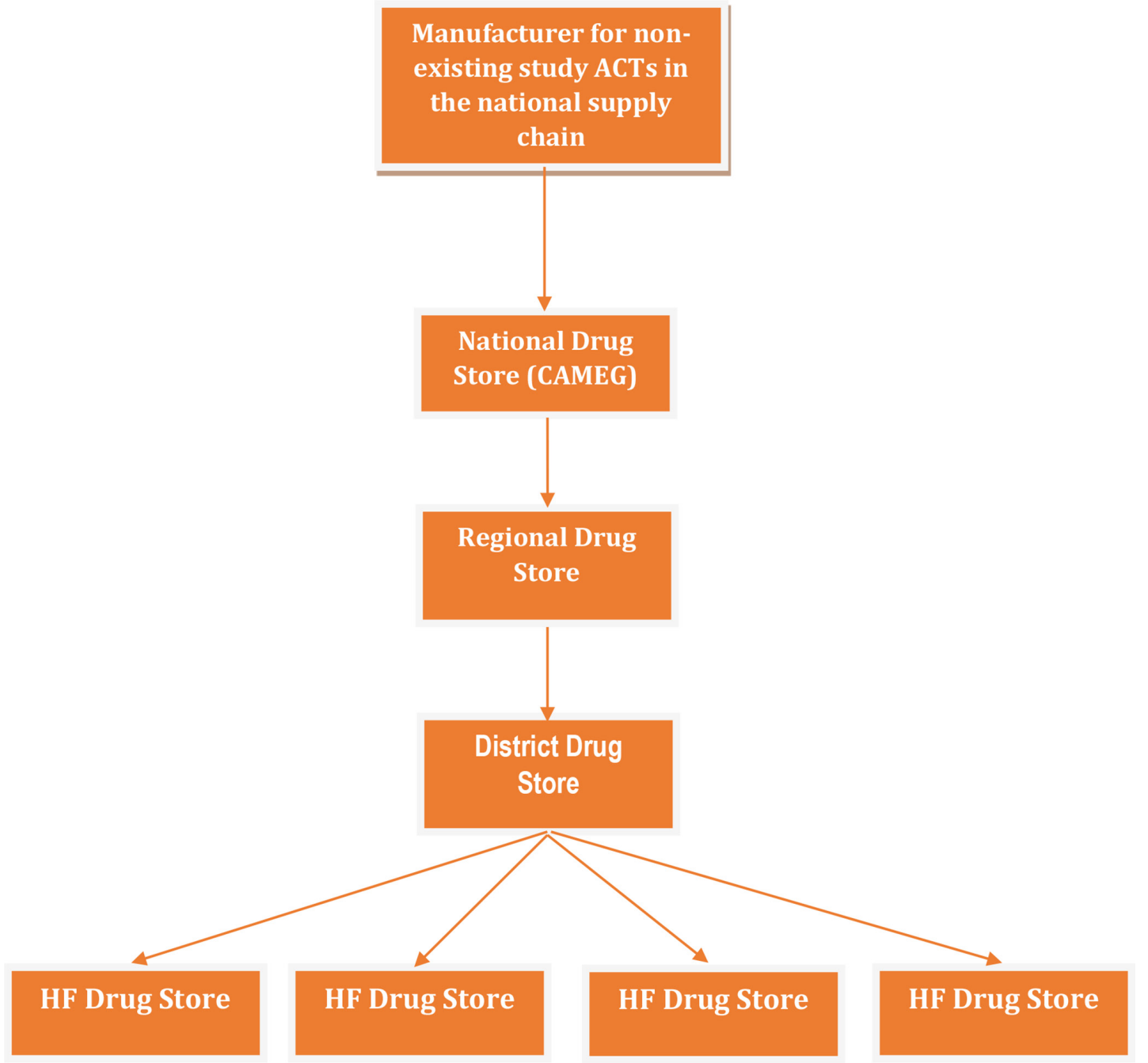

Figure 2 Artemisinin-based combination therapies flow in the framework of the multiple first-line therapies programme. ACT, artemisinin-based combination therapies; CAMEG, central essential drugs store.

- To promote health workers' adherence to the MFT protocol in the study communities.

\section{Monitoring and evaluation phase}

- General objective:

To assess the feasibility, acceptability, costs and the effects of the MFT programme for the management of uncomplicated malaria cases.

- Specific objectives

- To ensure continuous monitoring of the MFT deployment progress.

- To assess the implementation of the MFT for uncomplicated malaria.

- To determine the coverage of febrile episodes/ malaria promptly and appropriately managed by health workers (HWs).

- To determine the adherence of caregivers/febrile patients to ACT treatment regimens.

- To assess the acceptability of the MFT deployment strategy.

- To determine the costs of the MFT programme's implementation at district level.
- To assess the quality of care provided/performance of HWs.

- To assess the impact of the MFT programme on the morbidity and mortality rates related to febrile episodes/malaria.

- To assess the effects of the MFT programme on treatment-seeking behaviour for fever episodes/ malaria.

\section{Study outcomes}

The study outcomes, methods of assessment, time-points for assessment, targeted populations and data collections tools are summarised in online supplemental table S1.

\section{Sampling strategies}

Quantitative surveys

The true prevalence of fever episode/malaria at the community level in the programme settings and in the context of SMC is not well known. Assuming a prevalence ranging between $30 \%$ and $60 \%$ during the high transmission season of malaria before the deployment of the MFT programme, and a modest $10 \%$ reduction during MFT 
deployment, a sample size of 450 will be needed in each study group (children under 5, children 5-15 years of age and 16-40 years of age), with a $95 \%$ confidence level, $80 \%$ power and $10 \%$ rate of unforeseeable events.

There is no formal sample size calculation for pregnant women. These will be recruited exhaustively as fieldworkers identify them during the surveys at the community level. We will not perform any pregnancy testing at home. Pregnant women will be enrolled only with proof of ongoing pregnancy documented in their antenal care visit cards.

\section{Qualitative surveys}

The sampling strategy for the collection of the qualitative data is summarised in table 1 .

\section{Data collection approaches}

\section{Quantitative surveys}

\section{Household surveys}

A two-stage sampling process will be used. First, a sample of the targeted villages will be randomly selected from the list of all the villages in the Kaya health district. Within the selected villages, households will be randomly visited using a 'random walk' method. Children under five, pregnant women, individuals aged 5-15 years and individuals aged 16-40 years (adult population) will be invited to take part in the survey until the sample size for the village is reached. A household is defined as a family unit including the head of the household, his spouse (s) and other relatives living together and sharing their income.

A paper questionnaire will be used to collect information from the caregivers of children under five, pregnant women, individuals, aged 5-15 years and 16-40 years.

\section{Healthcare services utilisation}

Information on the utilisation of health facilities by the community will be collected from the health facility registers and through interviews of the target populations during the household surveys. The registers will provide a health facility utilisation rate, using as denominator the total number of individuals in the target age groups living in the study area obtained from the Kaya-HDSS. The interviews of target population will provide the coverage rate, eg, the proportion of fever episodes or confirmed malaria cases treated by the health workers according to the MFT programme. The numerator will be the total number of fever episodes or confirmed malaria cases that occurred during the four weeks preceding the household surveys, or the total number of cases of fever episodes/malaria seen at the health facility level.

\section{Update of Kaya-HDSS data}

An exhaustive assessment of malaria morbidity and mortality will be conducted using the updated Kaya-HDSS data, and the data generated one calendar year before the project will be compared to those generated during the year of MFT deployment.

\section{Qualitative surveys}

The qualitative surveys will be carried out by socials scientists (interviewers/facilitators) holding a Bachelor/Master degree in socioanthropology, under the supervision of a senior social scientist. They will be recruited through a call for applications after which they will receive a comprehensive training on the study's objectives, specific standard operating procedures, data collection tools and process of consent taking. The training sessions will be followed by preliminary field tests to identify and address any misunderstanding regarding the interview guides, data collection process, and to adapt the interview guides before the surveys begin.

\section{In-depth interviews}

In-depth individual interviews (IDI) of various respondents will be conducted, including heath system stakeholders (at central, intermediate and peripheral level), health system managers, healthcare providers and community members (key opinion leaders, pregnant women, mothers of children under five, and adult population). The IDI will aim to establish the range of perceptions on the MFT programme, and the sources of care, as well as provide an overview of the intervention. The interviews will be conducted in the local language or in the language best spoken by the participant and by trained social scientists using structured interview guides.

\section{Focus group discussion}

Focus group discussions (FGDs) will be held with household heads (male participants), mothers (female caregivers), and adult population members, potential beneficiaries of the intervention at the health facility level and HWs working in the programme catchment area. The aim will be to obtain data on perceptions of the MFT programme and perceptions of fever/malaria case and its management. The discussion will be conducted in the local language, or the language best spoken by the participants, using structured interview guides. The discussions will be recorded and then transcribed and translated into French for analysis. The FGDs will gather 8-12 participants per session.

\section{Assessment of the cost to the health service of offering the MFT programme}

The time spent on the MFT programme by HWs will be captured by asking them (possibly a sample) to keep a time log of their activities on the programme (diary). The value of their time will be determined from estimates of opportunity costs (minimum wage, the average salary of the area). The programme's accounting system will provide information on expenditures over the one and half year duration of the programme. Many cost items, such as programme personnel salaries, supplies, utilities and transport, as well as mRDTs and ACTs will be considered. A visit to the programme's offices will be conducted to make an inventory of capital goods, such as equipment, furniture, vehicles and offices. 
Table 1 Sampling strategy for qualitative surveys

\begin{tabular}{|c|c|c|}
\hline Target group & $\begin{array}{l}\text { Data collection methods } \\
\text { and sample size }\end{array}$ & Sampling strategy \\
\hline The National Malaria Control Programme & 3 IDI & $\begin{array}{l}\text { All personnel with a key role in malaria case management } \\
\text { strategy delivery }\end{array}$ \\
\hline $\begin{array}{l}\text { North-central health region (head of the } \\
\text { health region, lead pharmacist, responsible of } \\
\text { the disease control service) }\end{array}$ & $3 \mathrm{IDI}$ & $\begin{array}{l}\text { All personnel with a key role in malaria case management } \\
\text { strategy delivery }\end{array}$ \\
\hline $\begin{array}{l}\text { Heath district management team of Kaya } \\
\text { (head of the health district, lead pharmacist } \\
\text { responsible for the district-level healthcare } \\
\text { provision) }\end{array}$ & $3 \mathrm{IDI}$ & $\begin{array}{l}\text { All personnel with a key role in malaria case management } \\
\text { strategy delivery }\end{array}$ \\
\hline Heads of the local health facilities & 5 IDI, 4 FGD & $\begin{array}{l}5 \text { heads of the local health facilities in the Kaya health district will } \\
\text { be randomly selected and invited for the IDI. The } 40 \text { heads of } \\
\text { local health facilities will be allocated randomly into four groups } \\
\text { for the conduct of the FGD. }\end{array}$ \\
\hline Local health workers & 10 FGD & $\begin{array}{l}10 \text { local health facilities in the Kaya health district will be } \\
\text { randomly selected and the local health workers in charge of } \\
\text { malaria case management in pregnant women, children under } 5 \\
\text { and individuals of } 5 \text { years old and above, will be grouped for the } \\
\text { conduct of the FGD. }\end{array}$ \\
\hline Community key opinion leaders & 10 IDI, 4 FGD & $\begin{array}{l}10 \text { local health facilities in the Kaya health district will be } \\
\text { randomly selected, and the head of the health facilities will } \\
\text { appoint a community leader per their respective health area for } \\
\text { the conduct of the IDI. Subsequently, } 4 \text { community leaders per } \\
10 \text { health facility previously selected will be identified by the } \\
\text { head of the health facility to constitute the group for the conduct } \\
\text { of the FGD. }\end{array}$ \\
\hline
\end{tabular}

Heads of household (male participants) 5 FGD

During the household surveys, villages will be selected at random within the Kaya health district. Households will be selected at random via the random walk method, with the interval selected dependent on village size and the head of households (males) will be invited for the conduct of FGD.

Caregivers of children under 5 (female $\quad 5$ FGD
participants)

During the household surveys, villages will be selected at random within the Kaya health district. Caregivers (mothers of children under 5) will be selected at random via the random walk method, with the interval selected dependent on village size and will be invited for the conduct of FGD.

$\begin{array}{lll}\text { Pregnant women } & 5 \text { FGD } & \begin{array}{l}\text { During the household surveys, villages will be selected at } \\ \text { random within the Kaya health district. Pregnant women will } \\ \text { be selected at random via the random walk method, with the } \\ \text { interval selected dependent on village size and will be invited for } \\ \text { the conduct of FGD. }\end{array} \\ \text { Adult participants } & 5 \text { FGD } & \begin{array}{l}\text { During the household surveys, villages will be selected at } \\ \text { random within the Kaya health district of Kaya. Adult potential } \\ \text { participants will be selected at random via the random walk } \\ \text { method, with the interval selected dependent on village size and } \\ \text { will be invited for the conduct of FGD. }\end{array} \\ \end{array}$

\section{Total of interviews}

32 IDI and 42 FGD

ACT, artemisinin-based combination therapies; FGD, focus group discussion; IDI, in-depth individual interviews.

For personnel not directly involved in the intervention but still spending time on the programme, a questionnaire will be designed to capture data on their time spent during oral interviews at their workplace (health centres, regional offices...). Regarding the various preparatory activities during the formative phase, the research team will create and maintain an inventory of all the resources used for training workshops of HWs, 
and develop communication material and community outreach campaigns.

\section{Data management and analysis}

Quantitative data

Every month, summary data generated by health facilities will be collected by the field supervisors using a standard data extraction form and monitoring report template. These data will be shared with the district health office and a copy will be sent to the research institution. Poor quality data issues and other queries will be managed regularly rather than at the end of the study, to maximise data completeness and quality, and ensure that the final analysis is performed on time. All the data collected at both the health facility and the community levels will be checked before being double entered independently in the database.

Efforts will be made during the study to minimize and track missing data, as no attempt will be made to impute for missing data during the final analysis. All the data entered will be checked for inconsistencies and corrections will be made accordingly. Basic descriptive univariate and bivariate analysis will be performed to describe sociodemographic and economic characteristics of the study populations, as well as communities' knowledge, attitudes and practices regarding febrile episodes/malaria and access to effective malaria case management, including diagnostic and treatment before and during the MFT deployment. Appropriate statistics including proportions, means, medians, interquartile ranges, standard deviations and confidence intervals will be computed. The effect of MFT on treatment seeking behaviour for febrile episode/malaria and on the malaria incidence will be assessed by multivariate analysis using logistic regression and the approach of interrupted time series analysis, respectively. The effect of MFT on febrile episode/malariarelated mortality will be assessed using the mortality rate ratios derived from data collected one calendar year before and one calendar year along MFT deployment.

\section{Qualitative data}

Data management will begin during data collection by transcribing and translating each audio-recorded IDI and FGD and expanded notes when audio recordings are not available. All transcripts will be typed into a word processing programme and stored on password-protected computers. All participants will be assigned unique identification codes to facilitate data tracking and no identifiable information will be collected. Data management logs will be created to track and monitor data collection and transcription.

As soon as possible after the interviews have been conducted, each transcript will be read carefully by the study investigators in order to ${ }^{1}$ : ask any question from the text that may be unclear'; point out areas in which interviewing and transcription techniques could be improved and $^{3}$ identify recurrent themes and areas for future probing.

Data-derived codes developed through inductive coding and retrieving will be used during analysis. A priori codes for retrieving text for key concepts related to the overall objectives also will be applied to the data. Investigators will determine a coding frame to be used based on the topic guides and the first few transcripts available for analysis. New codes will be added as necessary during transcript analysis. The qualitative data software programme QSR NVivo will be used to organise all qualitative data and prepare them for analysis. Procedures will be put into place to check for intercoding discrepancies. Once all the transcripts have been coded, textual coding reports will be produced. Data reduction techniques will be used to examine codes in detail for subthemes and patterns across the transcripts.

\section{ETHICS AND DISSEMINATION}

The National Health Research Ethics Committee of Burkina Faso first approved the study protocol and informed consent documents (Clearance no. 2018-8-113). The approval is valid for oneyear and therefore must be renewed every year. The study will be performed in accordance with the ethical principles stated in the Declaration of Helsinki 1964, as revised in Fortaleza in 2013.

All participants in the different surveys of the formative and evaluation phases will be asked to voluntarily give written informed consent before any study-specific data collection. A copy of the informed consent form will be shared with each participant. Participant's full name will not be written on any data collection instruments and an identification code will be assigned to each participant. Participants' names will not be written in the transcripts of the interviews. A unique identification numbers will also be assigned to each participant during data analysis activities. All electronic files will be protected by a password and stored on password-protected computers. The copies of all the informed consent forms will be stored securely in a locked cabinet at the research institution offices, separately from study questionnaires or interview transcripts. Names will not be included in any formal or informal presentation, nor in any manuscript.

There are few anticipated risks associated with participation in the different surveys. Some participants may feel uncomfortable or embarrassed when asked questions about their current practices or their attitudes towards malaria case management. The research staff will make all the effort to protect individual participants' privacy and confidentiality. There are no direct benefits for those taking part in the various surveys. However, the information provided by the participants will be immediately used to inform the design of MFT programme, and will be useful later to generate evidence to support decision regarding the adoption of the MFT strategy for the management of uncomplicated malaria. 
Any protocol amendment will be agreed upon by the investigators and Medicine for Malaria Venture project management team in the form of a written amendment. Amended protocol will then be submitted for ethical clearance.

The findings of this study will be disseminated through feedback meetings for reporting to local communities, national workshop with researchers, policy-makers, and oral presentations at national and international congresses, conferences and seminars. We will also submit the results to peer-reviewed scientific journals for publications.

\section{PATIENT AND PUBLIC INVOLVEMENT}

Study participants have not been involved in the development of the research question, outcome measures, or design of the study. Likewise, they will not be also involved in the recruitment procedures. Data will be collected from households, study participants both at the community and at the health facility levels. Households in the study area will be randomly selected and potential subjects living within the selected households and who meet the eligibility criteria will be invited to participate in the study after obtaining informed consent. Study results will be disseminated to the study population through feedback meetings with the communities' leaders and representatives.

\section{DISCUSSION}

The MFT strategy has not yet been implemented in a structured way in sub-Saharan Africa. This study will be among the first to generate evidence on the feasibility, acceptability and cost of MFT stragtegy.

MFT is one of the potential strategies that could mitigate effectively the emergence and spread of artemisinin resistance, which pose a serious threat to malaria control and elimination. Indeed, the loss of artemisinin effectiveness would likely result in the loss of all ACTs. Therefore, there is urgent need to identify and implement in subSaharan Africa a better way to optimise the use of these ACTs, that could provide a much higher long-term barrier to the emergence and/or the spread of resistance, and thereby protecting them. Theoretical models showed that the simultaneous deployment of MFT for uncomplicated malaria is a promising strategy to extend the useful therapeutic life of the current ACTs by reducing drug pressure and slowing the spread of resistance without putting patients' lives at risk. It is therefore necessary to develop feasible strategies to implement MFT. ${ }^{23}{ }^{24}$ Treating half of the population with one ACT and the other half with another ACT is expected to reduce somewhat the fitness of resistant parasites while treating half as many patients. ${ }^{25}$ Therefore, there is an advantage in deploying two or more ACTs. There are several scenarios for the implementation of the MFT strategy, depending on the target: (1) breaking down the ACT target population into several segments: paediatric patients, pregnant women, adults patients, (2) using one ACT for home-based care and another ACT in healthcare facilities, (3) alternating different ACTs within the same population over a given period of time, etc. ${ }^{26}$

In this study, we opted for the segmentation of the overall population, a different ACT being allocated to each segment, based on the availability of the ACTs registered in the country. PYR-AS, AL and DHA-PQ will be assigned respectively to children under five, pregnant women as of their second trimester of pregnancy and individuals aged fiveyears and above. Indeed, AS-AQ was excluded due to the wide use of amodiaquine in combination with sulfadoxine-pyriméthamine for the SMC programme in Burkina Faso. In order to lower the pressure on amodiaquine and thus protect it, the NMCP in Burkina Faso is planning to remove it in the near future from its guidelines for the management of uncomplicated malaria. The choice of AL for pregnant women is justified by its effectiveness and its good safety profile in this vulnerable population. ${ }^{8} \mathrm{CCMm}$ strategy is also underway in the country using AL. Therefore, we aim to alleviate the pressure on AL by reducing its use at the health facility level and reserving it for a small proportion of the population (pregnant women). PYR-AS will be assigned to the children under five based on its good effectiveness and safety profile, ${ }^{4-627}$ the availability of a child friendly formulation which does not interact with food intake, and its easy dosage regimen (only one daily dose for three days) that can improve treatment compliance. Finally, DHA-PQ will be assigned to the rest of the population, ie, individuals aged five years and above. This drug also has been shown to be well-tolerated, efficacious and effective in preventing incident infections. ${ }^{5727}$ The absence of paediatric formulation prevents its use in children less than five.

Irrespective of the scenario used, the deployment of the MFT is expected to raise several challenges including setting up the the right distribution channel for the ACTs, managing the logistics, compensating the higher costs of some ACTs, adjusting the health system's drug taking into account less favoured drugs. A potential limitation of this study is that it will not examine the changes in molecular markers of ACTs resistance before and after the MFT deployment. Findings from this research are pivotal to inform stakeholders on the future of this strategy.

\section{Author affiliations}

${ }^{1}$ Département Biomédical et Santé Publique, Groupe de Recherche Action en Sante (GRAS), Ouagadougou, Burkina Faso

${ }^{2}$ Access and Product Management, Medecine for Malaria Venture, Geneva, Switzerland

${ }^{3}$ Programme National de Lutte contre le Paludisme, Ministère de la Santé, Ouagadougou, Burkina Faso

${ }^{4}$ Département Biomédical et Santé Publique, Institut de Recherche en Sciences de la Santé (IRSS), Kaya, Burkina Faso

${ }^{5}$ Département Biomédical et Santé Publique, Institut de Recherche en Sciences de la Sané (IRSS), Ouagadougou, Burkina Faso

Twitter Nouhoun Barry @Dr Barry Nouhoun and Adama Baguiya @baguiya 
Acknowledgements We thank colleagues from various institutions for constructive conversations and discussions as we developed the ideas. We also thank CAMEG for agreeing to become a partner of this study, responsible for the storage and distribution of the study ACTs through the official drug supply chain in Burkina Faso. Finally, we thank the Ministry of Health in Burkina Faso for the approval of this protocol and for procuring artemether-lumefantrine one of the study ACTs. We are grateful to Shin Poong Pharmaceuticals Co., Ltd. who provided PYR-AS for the study.

Contributors MS, A-MT, IS and SBS designed the study. MS, A-MT, IS, ABT and SBS developed the study protocol. YN, AKK, NB, AH, YS, SK participated in the finalisation of the protocol. MS wrote the first draft of the manuscript. All authors critically reviewed the manuscript and approved the final version of the manuscript.

Funding This work is supported by Medicine for Malaria Venture (MMV), grant number: P018/00879.

Competing interests None declared.

Patient consent for publication Not required.

Ethics approval Comité d'éthique pour la recherché en santé (CERS), Burkina Faso

Provenance and peer review Not commissioned, externally peer reviewed.

Supplemental material This content has been supplied by the author(s). It has not been vetted by BMJ Publishing Group Limited (BMJ) and may not have been peer-reviewed. Any opinions or recommendations discussed are solely those of the author(s) and are not endorsed by BMJ. BMJ disclaims all liability and responsibility arising from any reliance placed on the content. Where the content includes any translated material, BMJ does not warrant the accuracy and reliability of the translations (including but not limited to local regulations, clinical guidelines, terminology, drug names and drug dosages), and is not responsible for any error and/or omissions arising from translation and adaptation or otherwise.

Open access This is an open access article distributed in accordance with the Creative Commons Attribution Non Commercial (CC BY-NC 4.0) license, which permits others to distribute, remix, adapt, build upon this work non-commercially, and license their derivative works on different terms, provided the original work is properly cited, appropriate credit is given, any changes made indicated, and the use is non-commercial. See: http://creativecommons.org/licenses/by-nc/4.0/.

\section{ORCID iDs}

Mohamadou SIRIBIE http://orcid.org/0000-0001-5254-6548

Adama Baguiya http://orcid.org/0000-0003-1016-4896

\section{REFERENCES}

1 Sécretariat Général, Ministère de la Sante, Direction Générale des Etudes et des Statistiques Sectorielles BF. Annuaire statistique 2018, 2019.

2 Santé Mde, PNLP BF. Plan stratégique 2016-2020 de lutte contre Le paludisme Au Burkina Faso, 2015.

3 Santé Mdela, PNLP BF. Directives nationales de prise en charge Du paludisme Au Burkina Faso, 2017.

4 Sagara I, Beavogui AH, Zongo I, et al. Safety and efficacy of retreatments with pyronaridine-artesunate in African patients with malaria: a substudy of the WANECAM randomised trial. Lancet Infect Dis 2016;16:189-98.

5 West African Network for Clinical Trials of Antimalarial Drugs (WANECAM). Pyronaridine-artesunate or dihydroartemisininpiperaquine versus current first-line therapies for repeated treatment of uncomplicated malaria: a randomised, multicentre, open-label, longitudinal, controlled, phase 3b/4 trial. Lancet 2018;391:1378-90.

6 Duparc S, Borghini-Fuhrer I, Craft CJ, et al. Safety and efficacy of pyronaridine-artesunate in uncomplicated acute malaria: an integrated analysis of individual patient data from six randomized clinical trials. Malar J 2013;12:70.

7 Adjei A, Narh-Bana S, Amu A, et al. Treatment outcomes in a safety observational study of dihydroartemisinin/piperaquine (Eurartesim(®)) in the treatment of uncomplicated malaria at public health facilities in four African countries. Malar J 2016;15:43.

8 The PREGACT Study Group. Four artemisinin-based treatments in African pregnant women with malaria. N Engl J Med Overseas Ed 2016;374:913-27.

9 Noedl H, Se Y, Schaecher K, et al. Evidence of artemisinin-resistant malaria in Western Cambodia. N Engl J Med 2008;359:2619-20.

10 Dondorp AM, Nosten F, Yi P, et al. Artemisinin Resistance in Plasmodium falciparum Malaria. N Engl J Med Overseas Ed 2009;361:455-67.

11 World Health Organization GMP. Status report on artemisinin resistance [Internet], 2014. Available: https://www.who.int/malaria/ publications/atoz/status rep artemisinin resistance jan2014.pdf

12 Ariey F, Witkowski B, Amaratunga C, et al. A molecular marker of artemisinin-resistant Plasmodium falciparum malaria. Nature 2014;505:50-5.

13 Phyo AP, Nkhoma S, Stepniewska K, et al. Emergence of artemisininresistant malaria on the Western border of Thailand: a longitudinal study. Lancet 2012;379:1960-6.

14 Amaratunga C, Sreng S, Suon S, et al. Artemisinin-Resistant Plasmodium falciparum in Pursat Province, Western Cambodia: a parasite clearance rate study. Lancet Infect Dis 2012;12:851-8.

15 Kyaw MP, Nyunt MH, Chit K, et al. Reduced susceptibility of Plasmodium falciparum to artesunate in southern Myanmar. PLoS One 2013;8:e57689.

16 Hien TT, Thuy-Nhien NT, Phu NH, et al. In vivo susceptibility of Plasmodium falciparum to artesunate in Binh Phuoc Province, Vietnam. Malar J 2012;11:355. 26 oct.

17 Imwong M, Suwannasin K, Kunasol C, et al. The spread of artemisinin-resistant Plasmodium falciparum in the greater Mekong subregion: a molecular epidemiology observational study. Lancet Infect Dis 2017;17:491-7.

18 Thanh NV, Thuy-Nhien N, Tuyen NTK, et al. Rapid decline in the susceptibility of Plasmodium falciparum to dihydroartemisininpiperaquine in the South of Vietnam. Malar J 2017;16:27.

19 Aguiar AC, de Sousa LRF, Garcia CRS, et al. New molecular targets and strategies for antimalarial discovery. Curr Med Chem 2019;26:4380-402.

20 Dipanjan B, Shivaprakash G, Balaji O. Triple combination therapy and drug Cycling-Tangential strategies for Countering artemisinin resistance. Curr Infect Dis Rep 2017;19:25.

21 Curtis CF, Otoo LN. A simple model of the Build-up of resistance to mixtures of anti-malarial drugs. Trans $R$ Soc Trop Med Hyg 1986;80:889-92.

22 Kouanda S, Bado A, Yaméogo M, et al. The Kaya HDSS, Burkina Faso: a platform for epidemiological studies and health programme evaluation. Int J Epidemiol 2013;42:741-9.

23 Nguyen TD, Olliaro P, Dondorp AM, et al. Optimum population-level use of artemisinin combination therapies: a modelling study. Lancet Glob Health 2015;3:e758-66.

24 Smith DL, Klein EY, McKenzie FE, et al. Prospective strategies to delay the evolution of anti-malarial drug resistance: weighing the uncertainty. Malar J 2010;9:217.

25 Boni MF, Smith DL, Laxminarayan R. Benefits of using multiple first-line therapies against malaria. Proc Natl Acad Sci U S A 2008;105:14216-21.

26 Boni MF, White NJ, Baird JK. The community as the patient in malaria-endemic areas: Preempting drug resistance with multiple first-line therapies. PLoS Med 2016;13:e1001984.

27 Bassat $\mathrm{Q}$, Mulenga $\mathrm{M}$, Tinto $\mathrm{H}$, et al. Dihydroartemisinin-piperaquine and artemether-lumefantrine for treating uncomplicated malaria in African children: a randomised, non-inferiority trial. PLoS One 2009;4:e7871. 